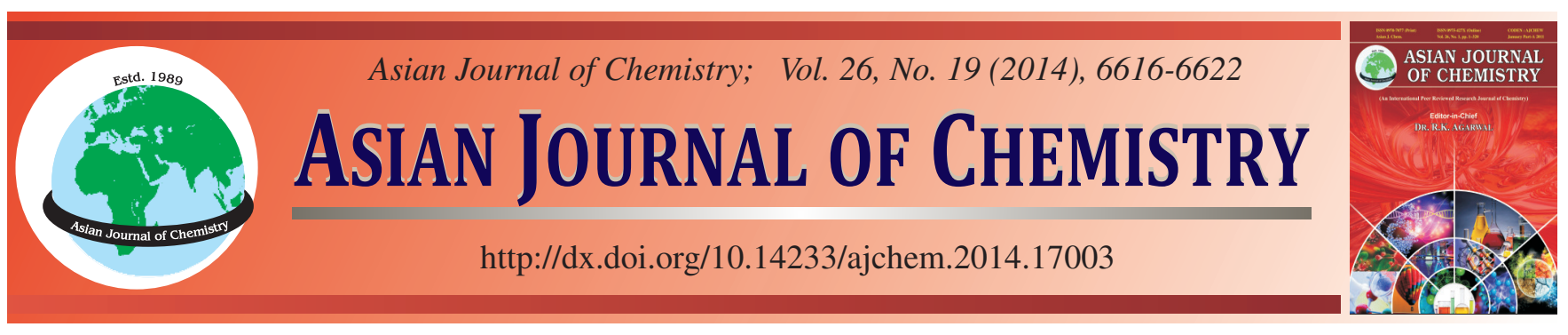

\title{
2-(3-Methyl-1-phenyl-1H-pyrazol-4-yl)-3-phenylthiazolidin-4-ones as Potent Antioxidant and Antidiabetic Agent
}

\author{
SANDIP SEN ${ }^{1, *}$, BIPLAB DE ${ }^{2}$ and T.S. EASWARI ${ }^{1}$
}

${ }^{1}$ IIMT College of Medical Sciences, O. Pocket, Ganga Nagar, Meerut-250 001, India

${ }^{2}$ Regional Institute of Pharmaceutical Science and Technology, Abhoynagar, Agartala-799 006, India

*Corresponding author: E-mail: sandipsen2010@gmail.com

Received: 7 January 2014;

Accepted: 22 February 2014;

Published online: 16 September 2014;

AJC-15974

\begin{abstract}
A series of 2-(3-methyl-1-phenyl-1H-pyrazol-4-yl)-3-phenylthiazolidin-4-ones (7a-7j) were synthesized by intramolecular cyclization of imines (6a-6j) with thioglycolic acid in the presence of acid catalyst. The Schiff bases were obtained upon reaction between electrophilic carbon atom of 3-methyl-1-phenyl-1H-pyrazole-4-carbaldehyde (4) and nucleophilic nitrogen atom of substituted aromatic amines. in vitro Antioxidant activity was determined by DPPH redical scavenging assay mehod using ascorbic acid as standard. The antidiabetic activity was carried out by streptazocine induced diabetic method using rosiglitazone as standard drug on wister rats. From the study it were found that 3-(4-chlorophenyl)-2-(3-methyl-1-phenyl-1H-pyrazol-4-yl)thiazolidin-4-one (7a), 3-(4-bromophenyl)-2-(3-methyl-1-phenyl-1Hpyrazol-4-yl)thiazolidin-4-one (7b), 3-(3,4-dichlorophenyl)-2-(3-methyl-1-phenyl-1H-pyrazol-4-yl)thiazolidin-4-one (7d), 3-(3,4difluorophenyl)-2-(3-methyl-1-phenyl-1H-pyrazol-4-yl)thiazolidin-4-one (7f) 2-(3-methyl-1-phenyl-1H-pyrazol-4-yl)-3-(4(trifluoromethyl) phenyl) thiazolidin-4-one (7h), 3-(4-methoxyphenyl)-2-(3-methyl-1-phenyl-1H-pyrazol-4-yl) thiazolidin-4-one (7j) shown more $\mathrm{IC}_{50}$ compare to standard. Where as compound $\mathbf{7 a}, \mathbf{7 b}, \mathbf{7 h}, \mathbf{7} \mathbf{j}$ show more significant antidiabetic effect against hyperglycemic rats compare to standard drugs at the dose of $5 \mathrm{mg} / \mathrm{kg}$ after 21 days of treatment.
\end{abstract}

Keywords: Pyrazole-4-carboxaldehydes, Thiazolidine-4-one, Antioxidant, Antidiabetic.

\section{INTRODUCTION}

Among the wide variety of heterocyclic compounds pyrazoles fused with different heterocycles known to contribute various pharmacological effects. Due to wide range of pharmacological activity there were significant amount of research activity directed towards this class. In particular, they were used as antitumor, antibacterial and antifungal, antiviral, antiparasitic, antitubercular and insecticidal agents. Some of these compounds have also shown antiinflammatory, antidiabetic, anesthetic and analgesic properties ${ }^{1}$. Another heterocyclic compound thiazolidine is a 5 membered saturated ring with a thio ether group at position 1 and an amino group at the position 3. It has fungicidal, local anesthetic, antiseizure, antitubercular, antibacterial, antiamoebic, antidiabetic and antiinflammatory activities $^{2,3}$. Thiazolidinones are derivatives of thiazolidine, which is a main pharmacophoric group responsible for antidiabetic activity. It acted by enhancing insulin sensitivity in both muscle and adipose tissue and to a lesser extent by inhibiting hepatic glucose production. These agents have a notable effect on improving insulin resistance, particularly when used in combination with other antidiabetic drugs, but have no effect on insulin secretion. As a class, the thiazolidinediones have also been shown to alter lipid profiles in patients with type 2 diabetes. They also have shown a decrease in triglyceride levels, increase in total and LDL cholesterol levels ${ }^{4}$. In patients receiving insulin therapy, the addition of a thiazolidinedione has resulted in significant reductions in daily insulin requirements ${ }^{5,6}$. The thiazolidine were dependent on the presence of insulin for activity; however, they do not affect insulin secretion. It was also found that patients with chronic pancreatitis had high risk of antioxidant deficiencies. Furthermore, this disease can lead to diabetes mellitus that could exacerbate the severity of oxidative stress. Oxidative stress and the resulting LDL oxidation considered major cause of atherosclerosis?

\section{EXPERIMENTAL}

All the chemicals used for the synthesis were reagent grade and of Sigma Aldrich and Merck Laboratory. The solvents were purified by standard laboratory procedure and free from atmospheric oxygen. The melting points were determined by open capillary method and were not corrected. The IR spectra were recorded in $\mathrm{KBr}$ pellets on a Shimadzu 8201 PC FTIR spectrophotometer. Both ${ }^{1} \mathrm{H}$ and ${ }^{13} \mathrm{C}$ NMR were recorded in DMSO- $d_{6}$ using Bruker $500 \mathrm{MHz}-\mathrm{NMR}$ spectrophotometers 
using TMS as internal standard. The masses of the compound were analyzed by ESI-mass method using Thermo Fennigan mass spectrophotometer. Elemental analyses were recorded using Thermo Finnigan FLASH EA 1112 CHN analyzer. TLC was performed in precoated plastic sheet of silica gel g/UV254 of $0.2 \mathrm{~mm}$ thickness.

Synthesis of 3-methyl-1-phenyl-1, 2-dihydropyrazol5-one (3): 1 Mole of phenyl hydrazine was taken in $500 \mathrm{~mL}$ beaker. 1 Mole equivalent of ethyl acetoacetate was added and allowed to warm the mixture at $100{ }^{\circ} \mathrm{C}$ on water bath for $3 \mathrm{~h}$. Cooling of the mixture at room temperature resulted solidification of crystal. Washing was taken place with ether and recrystallized from hot ethanol ${ }^{8}$. Yield: $93 \%$; m.p. 193-194 ${ }^{\circ} \mathrm{C} ;{ }^{1} \mathrm{H}$ NMR (DMSO- $d_{6}, \delta$ ppm): 7.18 (m, 2H, Ar-H), 6.716.69 (d, $1 \mathrm{H}, J=10 \mathrm{~Hz}, \mathrm{Ar}-\mathrm{H}), 6.66-6.64$ (d, 2H, $J=10 \mathrm{~Hz}$, Ar-H), 5.18 (s, 1H, Ar-H of pyrazole), 2.15 (s, 1H, -NH- of pyazole), 1.17 (s, 3H, $-\mathrm{CH}_{3}$ of pyrazole).

Synthesis of 3-methyl-1-phenyl-1H-pyrazole-4-carbaldehyde (4): Taken 3 mole of 3-methyl-1-phenyl-1,2-dihydropyrazol-5-one (3) in round bottom flask and was added 3 mole of DMF and 1 mole of $\mathrm{POCl}_{3}$. During addition of $\mathrm{POCl}_{3}$ the temperature of reaction mixture was maintained $0-5{ }^{\circ} \mathrm{C}$. After completion of addition reflux the mixture for $6 \mathrm{~h}$. Added sodium hydroxide and was cool for overnight. After addition of crushed ice formation of precipitation was taken place. Recrystallized from hot ethanol ${ }^{9}$. Yield: $83 \%$; m.p. $146-147{ }^{\circ} \mathrm{C}$; ${ }^{1} \mathrm{H}$ NMR (DMSO- $d_{6}, \delta$ ppm): 8.52 (s, 1H, -CHO), 7.82 (s, $1 \mathrm{H}, \mathrm{Ar}-\mathrm{H}$ ); 7.31 (s, 6H, Ar-H); 2.79 (s, 3H, $-\mathrm{CH}_{3}$ of pyrazole).

Synthesis of $N$-((3-methyl-1-phenyl-1H-pyrazol-4-yl) methylene) benzenamine (6) ${ }^{10}: 1 \mathrm{mmol}$ equivalent of 3-methyl-1-phenyl-1 $H$-pyrazole-4-carbaldehyde (4) was added in $50 \mathrm{~mL}$ of ethanol. Stirred the reaction mixture for $1 \mathrm{~h}$ under nitrogen atmosphere. Added $1 \mathrm{mmol}$ of glacial acetic acid. After that added substituent aromatic amines in $1 \mathrm{mmol}$ equivalent and refluxed for $1 \mathrm{~h}$. Then added crushed ice, filter the precipitate. Dried at room temperature and recrystallized from hot ethanol ${ }^{10}$.

4-Chloro- $N$-[(3-methyl-1-phenyl-1H-pyrazol-4-yl)methylene]benzenamine (6a): Yield: $93 \%$; m.p. 85-86 ${ }^{\circ} \mathrm{C}$; IR $\left(\mathrm{KBr}, v_{\max }, \mathrm{cm}^{-1}\right)$ : 2955.34(- $\left.\mathrm{CH}_{3}, \mathrm{str}\right), 1450.14\left(-\mathrm{CH}_{3}, \mathrm{def}\right)$, 1671.67 (C=N, str), 664.21 (C-Cl, str); ${ }^{1} \mathrm{H}$ NMR (DMSO- $d_{6}$, $\delta \mathrm{ppm}): 13.7$ (s, 1H, -NH ), $7.52(\mathrm{~s}, 1 \mathrm{H},-\mathrm{N}=\mathrm{CH}-), 7.31$ (s, $5 \mathrm{H}), 7.2-7.18$ (d, 2H, $J=10 \mathrm{~Hz}, \mathrm{Ar}-\mathrm{H}), 7.15$ (s, 1H, Ar-H), 6.8-6.78 (d, $2 \mathrm{H}, J=10 \mathrm{~Hz}, \mathrm{Ar}-\mathrm{H}) ;{ }^{13} \mathrm{C} \mathrm{NMR}$ (DMSO- $\left.d_{6}, \delta \mathrm{ppm}\right)$ : 160.1, 147.1, 145.5, 141.4,139.7, 132.8,131.4, 130.2, 130.2, 129.4, 129.4 126.3, 123.7, 123.7, 120.2, 105; ESI-Mass $(\mathrm{m} / \mathrm{z})$ : $295.09\left(\mathrm{M}^{+}\right)$. Anal. (\%) calcd. (found) for $\mathrm{C}_{17} \mathrm{H}_{14} \mathrm{~N}_{3} \mathrm{Cl}$ : C-69.03 (68.89), H- 4.77 (4.37), N-14.21(14.11).

4-Bromo- $\boldsymbol{N}$-[(3-methyl-1-phenyl-1H-pyrazol-4-yl)methylene]benzenamine (6b): Yield: $92 \%$; m.p. 85-86 ${ }^{\circ} \mathrm{C}$; IR $\left(\mathrm{KBr}, v_{\max }, \mathrm{cm}^{-1}\right): 2956.52\left(-\mathrm{CH}_{3}, \mathrm{str}\right), 1469.35\left(-\mathrm{CH}_{3}, \mathrm{def}\right)$, $1624.73(\mathrm{C}=\mathrm{N}, \mathrm{str}), 621.93(\mathrm{C}-\mathrm{Br}, \mathrm{str}) ;{ }^{1} \mathrm{H}$ NMR (DMSO- $d_{6}$, $\delta$ ppm): 13.7 (s, 1H, -NH ), 7.53 (s, 1H, -N=CH-), 7.21 (s, $5 \mathrm{H}), 7.19-7.18$ (d, 2H, J = $5 \mathrm{~Hz}, \mathrm{Ar}-\mathrm{H}), 7.15$ (s, 1H, Ar-H), 6.8-6.78 (d, $2 \mathrm{H}, J=10 \mathrm{~Hz}, \mathrm{Ar}-\mathrm{H}) ;{ }^{13} \mathrm{C} \mathrm{NMR}$ (DMSO- $\left.d_{6}, \delta \mathrm{ppm}\right)$ : 162.1, 147.5, 145.7, 141.4, 139.7, 132.8,131.4, 130.7,130.7, 129.4, 129.4, 126.3, 121.7, 121.7, 118.2, 105.7. ESI mass $(\mathrm{m} / \mathrm{z})$ : $339.13\left(\mathrm{M}^{+}\right)$; Anal. (\%) calcd. (found) for $\mathrm{C}_{17} \mathrm{H}_{14} \mathrm{~N}_{3} \mathrm{Br}$ : C-60.02 (59.89), H- 4.15 (4), N-12.35 (12.27).
4-Nitro- $N$-[(3-methyl-1-phenyl-1H-pyrazol-4-yl)methylene]benzenamine (6c): Yield: $87 \%$; m.p. 95-97 ${ }^{\circ} \mathrm{C}$; IR $\left(\mathrm{KBr}, v_{\max }, \mathrm{cm}^{-1}\right): 2953.52\left(-\mathrm{CH}_{3}, \mathrm{str}\right), 1685.73(\mathrm{C}=\mathrm{N}, \mathrm{str})$, $1540.02\left(\mathrm{C}-\mathrm{NO}_{2}\right.$, str), $1450.35\left(-\mathrm{CH}_{3}\right.$, def). ${ }^{1} \mathrm{H}$ NMR (DMSO$d_{6}, \delta$ ppm): 12.9 (s, 1H, -NH), 7.43 (s, 1H, -N=CH-), 7.33 (s, $5 \mathrm{H}), 7.20-7.18$ (d, 2H, $J=10 \mathrm{~Hz}, \mathrm{Ar}-\mathrm{H}), 7.15$ (s, 1H, Ar-H), 6.8-6.78 (d, 2H, $J=10 \mathrm{~Hz}$, Ar-H) ${ }^{13} \mathrm{C}$ NMR (DMSO- $d_{6}, \delta$ ppm): 162.1, 147.5, 145.2, 143.4, 136.7, 133.8, 131.4, 130.7, 130.7, 129.4, 129.4, 126.3, 121.7, 121.7, 118.2, 105.7; ESI mass $(\mathrm{m} / \mathrm{z})$ : $306.11\left(\mathrm{M}^{+}\right)$. Anal. (\%) calcd. (found) for $\mathrm{C}_{17} \mathrm{H}_{14} \mathrm{~N}_{4} \mathrm{O}_{2}$ : C-60.02 (59.89); H- 4.61 (4.41), N-18.21(18.19).

3, 4-Dichloro - $N$-[(3-methyl-1-phenyl-1H -pyrazol-4-yl)methylene]benzenamine (6d): Yield: $88 \%$; m.p. 83-84 ${ }^{\circ} \mathrm{C}$; IR $\left(\mathrm{KBr}, v_{\max }, \mathrm{cm}^{-1}\right): 2927.14\left(-\mathrm{CH}_{3}\right.$, str), $1434.24\left(-\mathrm{CH}_{3}\right.$, def $)$, 1679.67 (C=N, str), 764.21 (C-Cl, str); ${ }^{1} \mathrm{H}$ NMR (DMSO- $d_{6}$, $\delta$ ppm): 12.7(s, 1H, -NH), 7.53 (s, 1H, -N=CH-), 7.35 (s, 1H, Ar-H), 7.31 (s, 5H), 7.2-7.18 (d, 2H, J = 10 Hz, Ar-H), 7.117.09 (d, $1 \mathrm{H}, J=10 \mathrm{~Hz}, \mathrm{Ar}-\mathrm{H}) ;{ }^{13} \mathrm{C}$ NMR (DMSO- $\left.d_{6}, \delta \mathrm{ppm}\right)$ : 161.1, 148.5, 145.2, 143.3, 135.7, 132.8,131.4, 130.5, 130.5, 129.2, 129.2, 126.3, 121.7, 121.7, 118.2,105.7; ESI mass $(\mathrm{m} / \mathrm{z})$ : $329.02\left(\mathrm{M}^{+}\right)$; Anal. (\%) calcd. (found) for $\mathrm{C}_{17} \mathrm{H}_{13} \mathrm{~N}_{3} \mathrm{Cl}_{2}$ : C-61.83 (61.70), H- 3.97 (3.62), N-12.73(12.51).

4-Chloro- $\mathrm{N}$-((3-methyl-1-phenyl-1H-pyrazol-4-yl)methylene)-3-nitrobenzenamine (6e): Yield: $78 \%$; m.p. 93-

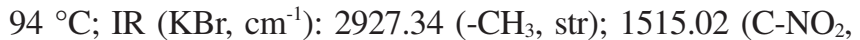
str); $1436.14\left(-\mathrm{CH}_{3}\right.$, def $), 1683.67(\mathrm{C}=\mathrm{N}, \mathrm{str}), 764.21$ (C-Cl, str); ${ }^{1} \mathrm{H}$ NMR (DMSO- $\left.d_{6}, \delta \mathrm{ppm}\right): 12.17(\mathrm{~s}, 1 \mathrm{H},-\mathrm{NH}), 7.43$ (s, 1H,$\mathrm{N}=\mathrm{CH}-), 7.37$ (s, 1H, Ar-H), $7.31(\mathrm{~S}, 5 \mathrm{H}), 7.21-7.18$ (d, 2H, $J=$ $15 \mathrm{~Hz}, \mathrm{Ar}-\mathrm{H}), 7.07-7.05$ (d, $1 \mathrm{H}, J=10 \mathrm{~Hz}, \mathrm{Ar}-\mathrm{H}) ;{ }^{13} \mathrm{C} \mathrm{NMR}$ (DMSO- $d_{6}, \delta$ ppm): 161.6, 148.7, 145.2, 143.3, 135.7, 132.7, 131.4, 130.3, 130.3, 129.1, 129.1, 126.3, 121.7, 121.7, 118.5, 105.3; ESI-MS ( $\mathrm{m} / \mathrm{e})$ : $340.07\left(\mathrm{M}^{+}\right)$. Anal. (\%) calcd. (found) for $\mathrm{C}_{17} \mathrm{H}_{13} \mathrm{~N}_{4} \mathrm{O}_{2} \mathrm{Cl}$ : C-59.92 (59.79), H-3.85 (3.81), N-16.44 (16.21).

3,4-Difluoro- $N$-[(3-methyl-1-phenyl-1H-pyrazol-4yl)methylene]benzenamine (6f): Yield- $68 \%$; m.p. $81-82{ }^{\circ} \mathrm{C}$; IR $\left(\mathrm{KBr}, v_{\max }, \mathrm{cm}^{-1}\right): 2925.14\left(-\mathrm{CH}_{3}, \mathrm{str}\right), 1534.34\left(\mathrm{C}-\mathrm{NO}_{2}\right.$, str), $1432.24\left(-\mathrm{CH}_{3}, \mathrm{def}\right), 1681.67(\mathrm{C}=\mathrm{N}, \mathrm{str}), 1363.21$ (C-F, str); ${ }^{1} \mathrm{H}$ NMR (DMSO- $\left.d_{6}, \delta \mathrm{ppm}\right): 13.17$ (s, $\left.1 \mathrm{H},-\mathrm{NH}\right), 7.41$ (s, $1 \mathrm{H},-\mathrm{N}=\mathrm{CH}-), 7.32$ (s, 1H, Ar-H), $7.3(\mathrm{~S}, 5 \mathrm{H}), 7.22-7.20$ (d, $2 \mathrm{H}, J=15 \mathrm{~Hz}, \mathrm{Ar}-\mathrm{H}), 7.17-7.15(\mathrm{~d}, 1 \mathrm{H}, J=10 \mathrm{~Hz}, \mathrm{Ar}-\mathrm{H}) ;{ }^{13} \mathrm{C}$ NMR (DMSO- $d_{6}, \delta$ ppm): $161.5,148.5,145.1,143.2,135.5$, 132.1, 131.1, 130.7, 130.7, 129.1, 129.1, 126.3, 121.7, 121.7, 118.5,105.4; ESI-MS (m/e): $297.07\left(\mathrm{M}^{+}\right)$; Anal. (\%) calcd. (found) for $\mathrm{C}_{17} \mathrm{H}_{13} \mathrm{~N}_{3} \mathrm{~F}_{2}$ : C-68.68 (68.28), H-4.41 (3.51), N14.13 (14.12).

2-Fluoro- $N$-[(3-methyl-1-phenyl-1H-pyrazol-4yl)methylene]-5-nitrobenzenamine (6g): Yield-79 \%; m.p. 93-94 ${ }^{\circ} \mathrm{C}$; IR $\left(\mathrm{KBr}, v_{\max }, \mathrm{cm}^{-1}\right)$ : $2927.14\left(-\mathrm{CH}_{3}, \mathrm{str}\right) ; 1534.34$ $\left(\mathrm{C}-\mathrm{NO}_{2}\right.$, str) $1433.24\left(-\mathrm{CH}_{3}\right.$, def); 1680.67 (C=N, str); 1160.21 (C-F, str). ${ }^{1} \mathrm{H}$ NMR (DMSO- $d_{6}, \delta$ ppm): 13.5 (s, $\left.1 \mathrm{H},-\mathrm{NH}\right)$, 8.2-8.18 (d, 2H, J = $10 \mathrm{~Hz}, \mathrm{Ar}-\mathrm{H}), 7.75$ (s, 1H, -N=CH-), 7.45 (s, 1H, Ar-H), 7.34 (S, 5H), 7.21-7.19 (d, 2H, J=10 Hz, Ar$\mathrm{H}) ;{ }^{13} \mathrm{C}$ NMR (DMSO- $d_{6}, \delta$ ppm): $163.5,149.5,146.1,143.7$, 135.7, 132.7, 131.1, 130.7, 130.7, 129.1, 129.1, 126.3, 121.5, 121.5, 118.5, 105.4; ESI-MS (m/e): $324.07\left(\mathrm{M}^{+}\right)$; Anal. (\%) calcd. (found) for $\mathrm{C}_{17} \mathrm{H}_{13} \mathrm{~N}_{4} \mathrm{O}_{2} \mathrm{~F}$ : C-62.96 (62.81), H- 4.04 (4.26), N-17.28 (17.11).

N-[(3-methyl-1-phenyl-1H-pyrazol-4-yl)methylene]-4(trifluoromethyl)benzenamine (6h): Yield: $95 \%$; m.p. 101- 
$102{ }^{\circ} \mathrm{C}$; IR $\left(\mathrm{KBr}, \mathrm{v}_{\max }, \mathrm{cm}^{-1}\right): 2927.14\left(-\mathrm{CH}_{3}\right.$, str $), 1433.24$ $\left(-\mathrm{CH}_{3}\right.$, def $), 1670.67(\mathrm{C}=\mathrm{N}, \mathrm{str}), 1136.21(\mathrm{C}-\mathrm{F}, \mathrm{str}) ;{ }^{1} \mathrm{H}$ NMR (DMSO- $d_{6}, \delta$ ppm): 13.7(s, 1H, -NH), 7.49 (s, 1H, -N=CH-), 7.37 (s, 5H), 7.21-7.18 (d, 2H, J=15 Hz, Ar-H), 7.15 (s, 1H, Ar-H), 6.8-6.78 (d, 2H, J = $10 \mathrm{~Hz}, \mathrm{Ar}-\mathrm{H}) ;{ }^{13} \mathrm{C}$ NMR (DMSO$\left.d_{6}, \delta \mathrm{ppm}\right): 163.5,149.5,146.1,143.7,135.7,132.7,131.1$, 130.7, 130.7, 129.1, 129.1, 126.3, 124.7, 121.5, 121.5, 118.5, 105.4; ESI-MS (m/e): $329.01\left(\mathrm{M}^{+}\right)$; Anal. (\%) calcd. (found) for $\mathrm{C}_{18} \mathrm{H}_{14} \mathrm{~N}_{3} \mathrm{~F}_{3}$ : C-65.65 (65.32), H-4.28 (3.39), N-12.76 (12.49).

N-[(3-methyl-1-phenyl-1H-pyrazol-4-yl)methylene]-4nitro-3-(trifluoromethyl)benzenamine (6i): Yield-78\%; m.p. $108-110{ }^{\circ} \mathrm{C}$; IR $\left(\mathrm{KBr}, v_{\max }, \mathrm{cm}^{-1}\right): 2935.24\left(-\mathrm{CH}_{3}, \mathrm{str}\right), 1413.02$ $\left(\mathrm{C}-\mathrm{NO}_{2}\right.$, str), 1336.24 (-CH 3 , def), 1640.67 (C=N, str), 1330.21 (C-F, str); ${ }^{1} \mathrm{H}$ N MR (DMSO- $d_{6}, \delta$ ppm): 13.1 (s, $\left.1 \mathrm{H},-\mathrm{NH}\right)$, 8.12-8.10 (d, 1H, $J=10 \mathrm{~Hz}, \mathrm{Ar}-\mathrm{H}), 7.7$ (s, 1H, Ar-H), 7.627.60 (d, 1H, J = $10 \mathrm{~Hz}, \mathrm{Ar}-\mathrm{H}), 7.49$ (s, 1H, -N=CH-), 7.31 (S, $5 \mathrm{H}), 7.25$ (s, $1 \mathrm{H}, \mathrm{Ar}-\mathrm{H}) ;{ }^{13} \mathrm{C}$ NMR (DMSO- $\left.d_{6}, \delta \mathrm{ppm}\right): 162.5$, 149.3, 145.1, 142.7, 135.3, 132.7, 131.1, 130.2, 130.2, 129.3, 129.3, 126.3, 124.7, 121.5, 121.5, 118.5,105; ESI-MS (m/e): $374.02\left(\mathrm{M}^{+}\right)$; Anal. (\%) calcd. (found) for $\mathrm{C}_{18} \mathrm{H}_{13} \mathrm{~N}_{4} \mathrm{O}_{2} \mathrm{~F}_{3}$ : C57.76 (57.46), H- 4.28 (3.39), N-14.97(14.59).

4-Methoxy- $\mathrm{N}$-[(3-methyl-1-phenyl-1H-pyrazol-4-yl) methylene]benzenamine (6j): Yield-78 \%; m.p. 95-96 ${ }^{\circ} \mathrm{C}$; IR $\left(\mathrm{KBr}, \mathrm{v}_{\max }, \mathrm{cm}^{-1}\right): 2824.14\left(-\mathrm{CH}_{3}, \mathrm{str}\right), 2815.22\left(-\mathrm{OCH}_{3}\right)$, $1432.24\left(-\mathrm{CH}_{3}\right.$, def), 1683.67 (C=N, str); ${ }^{1} \mathrm{H}$ NMR (DMSO$\left.d_{6}, \delta \mathrm{ppm}\right):$ 13.7(s, $\left.1 \mathrm{H},-\mathrm{NH}\right), 7.49$ (s, $\left.1 \mathrm{H},-\mathrm{N}=\mathrm{CH}-\right), 7.35$ (S, $5 \mathrm{H}), 7.21-7.18$ (d, 2H, J = $15 \mathrm{~Hz}, \mathrm{Ar}-\mathrm{H}), 7.15$ (s, 1H, Ar-H), 6.8-6.78 (d, 2H, $J=10 \mathrm{~Hz}, \mathrm{Ar}-\mathrm{H}), 3.73(\mathrm{~s}, 3 \mathrm{H}) ;{ }^{13} \mathrm{C} \mathrm{NMR}$ (DMSO- $d_{6}, \delta$ ppm): $163.5,149.5,146.1,143.7,135.7,132.7$, 131.1, 130.7, 130.7, 129.1, 129.1, 126.3, 121.5, 121.5, 118.5, 105.4, 55.9; ESI-MS (m/e): $291.17\left(\mathrm{M}^{+}\right)$; Anal. (\%) calcd. (found) for $\mathrm{C}_{18} \mathrm{H}_{17} \mathrm{~N}_{3} \mathrm{O}$ : C-74.20 (74.11), H-5.89 (5.62), N14.42 (14).

Synthesis of 2-(3-methyl-1-phenyl-1H-pyrazol-4-yl)-3phenylthiazolidin-4-one (7a-7j): The substituted imines $(0.02$ mole) were dissolved in toluene, $\mathrm{SnCl}_{2}(0.05 \mathrm{mmol})$ was used as catalyst. After addition of thioglycollic acid ( 0.03 mole) reaction mixtures were stirred for an hour. The mixtures were then refluxed in water bath for $12 \mathrm{~h}$ and cooled at room temperature (Scheme-I). Excess solvent were removed by distillation and compounds were dissolved in dichloromethane. The organic layer were washed with $10 \%$ sodium-bicarbonate and finally with brine solutions, dried over sodium sulphate and evaporated to dryness. Purification of the compounds were done with petroleum ether and chloroform $(2: 8 \mathrm{v} / \mathrm{v})$ by chromatographic technique.

3-(4-Chlorophenyl)-2-(3-methyl-1-phenyl-1H-pyrazol-4yl)thiazolidin-4-one (7a): Yield-68 \%; m.p. 143-144 ${ }^{\circ} \mathrm{C}$; IR $\left(\mathrm{KBr}, v_{\max }, \mathrm{cm}^{-1}\right)$ : $2959.34\left(-\mathrm{CH}_{3}, \mathrm{str}\right), 1775.67$ (C=O, str), 1455.14 (- $\mathrm{CH}_{3}$, def), 1260.33 (C-N, str), 760.21 (C-Cl, str); ${ }^{1} \mathrm{H}$ NMR (DMSO- $d_{6}, \delta$ ppm): 13.5 (s, 1H, -NH), 7.33 (s, 5H), 7.27.18 (d, 2H, J = $10 \mathrm{~Hz}, \mathrm{Ar}-\mathrm{H}), 7.15$ (s, 1H, Ar-H), 6.8-6.78 (d, $2 \mathrm{H}, J=10 \mathrm{~Hz}, \mathrm{Ar}-\mathrm{H}$ ), 4.85 (s, 1H, CH-N), 3.49 (s, 1H, $\mathrm{CH}_{2^{-}}$ $\mathrm{C}=\mathrm{O}), 3.24$ (s, $\left.1 \mathrm{H}, \mathrm{CH}_{2}-\mathrm{C}=\mathrm{O}\right) ;{ }^{13} \mathrm{C}$ NMR (DMSO- $d_{6}, \delta \mathrm{ppm}$ ): $170.9,147.1,145.5,141.4,139.7,132.8,131.4,130.2,130.2$, 129.4, 129.4, 126.3, 123.7, 123.7, 120.2, 105, 45, 45, 11; ESIMS (m/e): $369.17\left(\mathrm{M}^{+}\right)$; Anal. (\%) calcd. (found) for $\mathrm{C}_{19} \mathrm{H}_{16} \mathrm{~N}_{3} \mathrm{OSCl}$ : C-61.70 (61.66), H-4.36 (4.19), N-11.36(11.12).
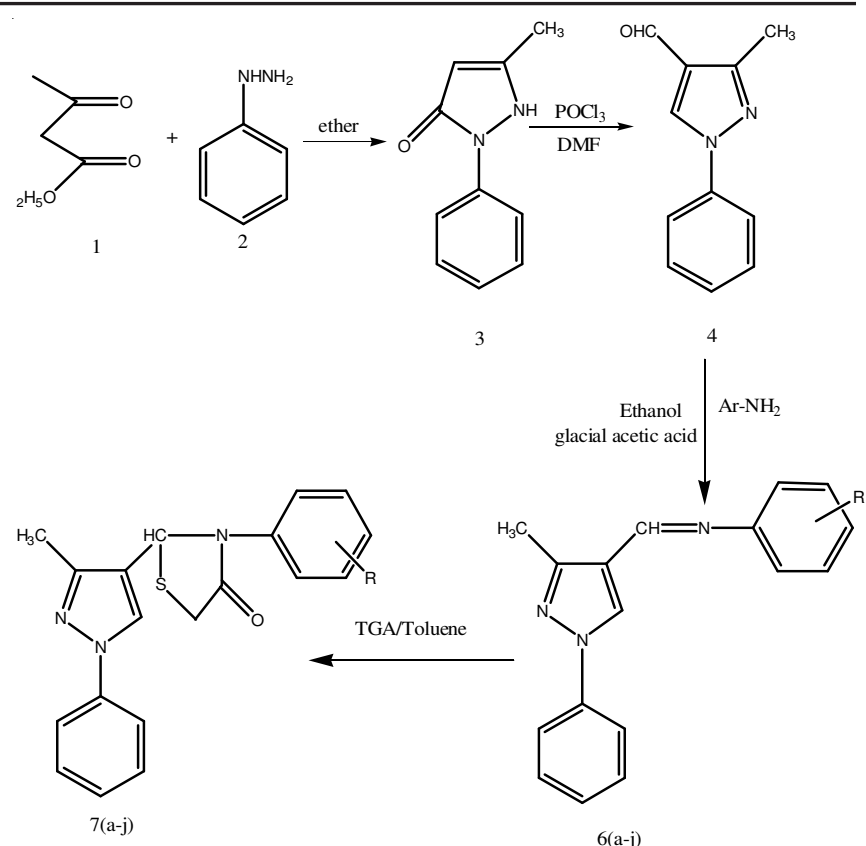

\begin{tabular}{llllll} 
Entry & \multicolumn{1}{c}{$\mathrm{R}$} & Entry & \multicolumn{1}{c}{$\mathrm{R}$} & Entry & \multicolumn{1}{c}{$\mathrm{R}$} \\
6a,7a & 4-chloro & $6 \mathrm{e}, 7 \mathrm{e}$ & 3-nitro-4-chloro & $6 \mathrm{~h}, 7 \mathrm{~h}$ & 4-trifluromethyl \\
6b,7b & 4-bromo & 6f,7f & 3,4-difluoro & $6 \mathrm{i}, 7 \mathrm{i}$ & 3-trifluoro-4-nitro \\
6c,7c & 4-nitro & $6 \mathrm{~g}, 7 \mathrm{~g}$ & 2-fluoro-5-nitro & $6 \mathrm{j}, 7 \mathrm{j}$ & 4-methoxy \\
6d,7d & 3,4-dichloro & & & &
\end{tabular}

Scheme-I

3-(4-Bromophenyl)-2-(3-methyl-1-phenyl-1H-pyrazol4-yl) thiazolidin-4-one (7b): Yield-66 \%; m.p. 133-134 ${ }^{\circ} \mathrm{C}$; IR $\left(\mathrm{KBr}, \mathrm{v}_{\max }, \mathrm{cm}^{-1}\right)$ : $2953.24\left(-\mathrm{CH}_{3}\right.$, str), 1692.61 (C=O, str), 1463.14 (- $\mathrm{CH}_{3}$, def), 1310.33 (C-N, str), 667.21 (C-Br, str); ${ }^{1} \mathrm{H}$ NMR (DMSO- $\left.d_{6}, \delta \mathrm{ppm}\right): 12.5$ (s, $\left.1 \mathrm{H},-\mathrm{NH}\right), 7.31$ (s, 5H), 7.21-7.19 (d, 2H, J = $10 \mathrm{~Hz}, \mathrm{Ar}-\mathrm{H}), 7.17$ (s, 1H, Ar-H), 6.816.79 (d, 2H, J = $10 \mathrm{~Hz}, \mathrm{Ar}-\mathrm{H}), 4.81$ (s, 1H, CH-N), 3.47 (s, $\left.1 \mathrm{H}, \mathrm{CH}_{2}-\mathrm{C}=\mathrm{O}\right), 3.22$ (s, 1H, $\left.\mathrm{CH}_{2}-\mathrm{C}=\mathrm{O}\right), 2.70$ (s, 3H, $-\mathrm{CH}_{3}$ ); ${ }^{13} \mathrm{C}$ NMR (DMSO- $\left.d_{6}, \delta \mathrm{ppm}\right): 171.9,147.2,146.5,142.4$, 139.5, 132.7, 131.3, 130.1, 130.1, 129.3, 129.3, 126.7, 123.8, 123.8, 120.1, 105.3, 45.1, 45.1, 11; ESI-MS (m/e): 415.02 $\left(\mathrm{M}^{+}\right)$; Anal. (\%) calcd. (found) for $\mathrm{C}_{13} \mathrm{H}_{12} \mathrm{~N}_{3} \mathrm{OSBr}$ : C-55.08 (54.96), H- 3.89 (3.69), N-10.14 (9.92).

2-(3-Methyl-1-phenyl-1H-pyrazol-4-yl)-3-(4-nitrophenyl)thiazolidin-4-one (7c): Yield-76\%; m.p. 138-139 ${ }^{\circ} \mathrm{C}$; IR $\left(\mathrm{KBr}, \mathrm{cm}^{-1}\right): 2949.24\left(-\mathrm{CH}_{3}, \mathrm{str}\right), 1701.61(\mathrm{C}=\mathrm{O}, \mathrm{str})$, $1525.02\left({\mathrm{C}-\mathrm{NO}_{2}}_{2}\right.$, str), 1461.34 (-CH $\mathrm{CH}_{3}$, def), 1321.33 (C-N, str); ${ }^{1} \mathrm{H}$ NMR (DMSO- $\left.d_{6}, \delta \mathrm{ppm}\right): 13.7(\mathrm{~s}, 1 \mathrm{H},-\mathrm{NH}), 7.31(\mathrm{~s}, 5 \mathrm{H})$, 7.21-7.21 (d, 2H, J=10 Hz, Ar-H), 7.15 (s, 1H, Ar-H), 6.826.80 (d, 2H, J=10 Hz, Ar-H), 4.85 (s, 1H, CH-N), 3.43 (s, $1 \mathrm{H}, \mathrm{CH}_{2}-\mathrm{C}=\mathrm{O}$ ), $3.22\left(\mathrm{~s}, 1 \mathrm{H}, \mathrm{CH}_{2}-\mathrm{C}=\mathrm{O}\right.$ ); ${ }^{13} \mathrm{C}$ NMR (DMSO$d_{6}, \delta$ ppm): 171.3, 147.1, 146.3, 141.4, 138.5, 132.5, 131.4, $130.5,130.5,129.5,129.5,127.7,124.8,124.8,120.1,105.3$, 45.3, 45.3, 11.1; ESI-MS (m/e): $380.09\left(\mathrm{M}^{+}\right)$; Anal. (\%) calcd. (found) for $\mathrm{C}_{19} \mathrm{H}_{16} \mathrm{~N}_{4} \mathrm{O}_{3} \mathrm{~S}$ : C-59.99 (59.70), H-4.24 (4.49), N14.74 (14.51).

3-(3,4-Dichlorophenyl)-2-(3-methyl-1-phenyl-1Hpyrazol-4-yl)thiazolidin-4-one (7d): Yield-68 \%; m.p. 110$111{ }^{\circ} \mathrm{C}$; IR $\left(\mathrm{KBr}, v_{\max }, \mathrm{cm}^{-1}\right): 2948.24\left(-\mathrm{CH}_{3}\right.$, str), 1720.61 (C=O, str), 1471.14 (- $\left.\mathrm{CH}_{3}, \mathrm{def}\right), 1321.33$ (C-N, str), 764.21 (C-Cl, str); ${ }^{1} \mathrm{H}$ NMR (DMSO- $d_{6}, \delta$ ppm): 12.7 (s, $\left.1 \mathrm{H},-\mathrm{NH}\right)$, 7.45 (s, 1H, Ar-H), 7.33 (s, 5H), 7.2-7.18 (d, 2H, J=10 Hz, 
Ar-H), 7.11-7.09 (d, 1H, J = $10 \mathrm{~Hz}, \mathrm{Ar}-\mathrm{H}), 4.83$ (s, 1H, CH$\mathrm{N}), 3.41\left(\mathrm{~s}, 1 \mathrm{H}, \mathrm{CH}_{2}-\mathrm{C}=\mathrm{O}\right) ; 3.22\left(\mathrm{~s}, 1 \mathrm{H}, \mathrm{CH}_{2}-\mathrm{C}=\mathrm{O}\right) ;{ }^{13} \mathrm{C} \mathrm{NMR}$ (DMSO- $d_{6}, \delta$ ppm): $170.3,146.1,143.3,141.2,133.5,132.7$, $131.3,130.2,130.2,129.5,129.5,126.7,123.8,123.8,120.1$, 108.3, 47.3, 47.3, 11.1; ESI-MS (m/e): $403.15\left(\mathrm{M}^{+}\right)$; Anal. (\%) calcd. (found) for $\mathrm{C}_{19} \mathrm{H}_{15} \mathrm{~N}_{3} \mathrm{OSCl}_{2}$ : C-56.44 (56.12), H3.74 (3.42), N-10.39(10.11).

3-(4-Chloro-3-nitrophenyl)-2-(3-methyl-1-phenyl-1Hpyrazol-4-yl)thiazolidin-4-one (7e): Yield-63 \%; m.p. 149$150{ }^{\circ} \mathrm{C}$; IR $\left(\mathrm{KBr}, v_{\max }, \mathrm{cm}^{-1}\right): 2948.24\left(-\mathrm{CH}_{3}, \mathrm{str}\right), 1720.61$ $(\mathrm{C}=\mathrm{O}, \mathrm{str}), 1525.02\left(\mathrm{C}-\mathrm{NO}_{2}, \mathrm{str}\right), 1471.14\left(-\mathrm{CH}_{3}, \mathrm{def}\right), 1321.33$ (C-N, str), 764.21 (C-Cl, str); ${ }^{1} \mathrm{H}$ NMR (DMSO- $d_{6}, \delta$ ppm): 12.3 (s, 1H, -NH), 7.43 (s, 5H), 7.35 (s, 1H, Ar-H), 7.2-7.18 $(\mathrm{d}, 2 \mathrm{H}, J=10 \mathrm{~Hz}, \mathrm{Ar}-\mathrm{H}), 7.11-7.09$ (d, 1H, $J=10 \mathrm{~Hz}, \mathrm{Ar}-\mathrm{H})$, 4.85 (s, 1H, CH-N), 3.49 (s, 1H, $\left.\mathrm{CH}_{2}-\mathrm{C}=\mathrm{O}\right), 3.25$ (s, $1 \mathrm{H}, \mathrm{CH}_{2}-$ $\mathrm{C}=\mathrm{O}$ ); ${ }^{13} \mathrm{C}$ NMR (DMSO- $d_{6}, \delta \mathrm{ppm}$ ): 170.3, 146.1, 143.3, 141.2, 133.5, 132.7, 131.3, 130.2, 130.2, 129.5, 129.5, 126.7, 123.8, 123.8, 120.1, 108.3, 47.3, 47.3, 11.1; ESI-MS (m/e): $414.08\left(\mathrm{M}^{+}\right)$; Anal. (\%) calcd. (found) for $\mathrm{C}_{19} \mathrm{H}_{15} \mathrm{~N}_{4} \mathrm{O}_{3} \mathrm{SCl}$ : C55.01 (54.80), H- 3.64 (3.45), N-13.50(13.11).

3-(3,4-Difluorophenyl)-2-(3-methyl-1-phenyl-1Hpyrazol-4-yl)thiazolidin-4-one (7f): Yield: $63 \%$; m.p. 163$164{ }^{\circ} \mathrm{C}$; IR $\left(\mathrm{KBr}, \mathrm{cm}^{-1}\right)$ : $2948.24\left(-\mathrm{CH}_{3}\right.$, str), $1720.61(\mathrm{C}=\mathrm{O}$, str), 1471.14 (- $\mathrm{CH}_{3}$, def), 1321.33 (C-N, str), 1201 (C-F, str); ${ }^{1} \mathrm{H}$ NMR (DMSO- $\left.d_{6}, \delta \mathrm{ppm}\right): 12.37(\mathrm{~s}, 1 \mathrm{H},-\mathrm{NH}), 7.32(\mathrm{~s}, 1 \mathrm{H}$, Ar-H), 7.29 (s, 5H), 7.23-7.21 (d, 2H, $J=10 \mathrm{~Hz}$, Ar-H), 7.117.09 (d, 1H, J = $10 \mathrm{~Hz}$, Ar-H), 4.83 (s, 1H, CH-N), 3.48 (s, $1 \mathrm{H}, \mathrm{CH}_{2}-\mathrm{C}=\mathrm{O}$ ), 3.15 (s, 1H, $\mathrm{CH}_{2}-\mathrm{C}=\mathrm{O}$ ); ${ }^{13} \mathrm{C}$ NMR (DMSO$\left.d_{6}, \delta \mathrm{ppm}\right): 171.3,145.1,143.7,141.3,135.5,133.7,131.8$, $130.2,130.2$, 127.5, 127.5, 125.7, 121.8, 121.8, 120.1, 108.3, 45.3, 45.3, 11.4; ESI-MS (m/e): $371.12\left(\mathrm{M}^{+}\right)$; Anal. (\%) calcd. (found) for $\mathrm{C}_{19} \mathrm{H}_{15} \mathrm{~N}_{3} \mathrm{OSF}_{2}$ : C-61.44 (61.11), H- 4.07 (3.82), N-11.31 (11.11).

3-(2-Fluoro-5-nitrophenyl)-2-(3-methyl-1-phenyl-1Hpyrazol-4-yl) thiazolidin-4-one (7g): Yield: $69 \%$; m.p. 131$132{ }^{\circ} \mathrm{C}$; IR $\left(\mathrm{KBr}, \mathrm{n}_{\max }, \mathrm{cm}^{-1}\right): 2927.14\left(-\mathrm{CH}_{3}, \mathrm{str}\right), 1720.61$ $(\mathrm{C}=\mathrm{O}, \mathrm{str}), 1534.34\left(\mathrm{C}-\mathrm{NO}_{2}, \mathrm{str}\right), 1321.33$ (C-N, str), 1433.24 $\left(-\mathrm{CH}_{3}\right.$, def), 1160.21 (C-F, str); ${ }^{1} \mathrm{H}$ NMR (DMSO- $\delta_{6}, \delta$ ppm): 13.5 (s, 1H, -NH), 8.2-8.18 (d, 2H, J=10 Hz, Ar-H), 7.45 (s, $1 \mathrm{H}, \mathrm{Ar}-\mathrm{H}), 7.39$ (s, 5H), 7.21-7.19 (d, 2H, $J=10 \mathrm{~Hz}, \mathrm{Ar}-\mathrm{H})$, 4.81 (s, 1H, CH-N), 3.43 (s, 1H, $\left.\mathrm{CH}_{2}-\mathrm{C}=\mathrm{O}\right) ; 3.15$ (s, $1 \mathrm{H}, \mathrm{CH}_{2}-$ $\mathrm{C}=\mathrm{O}) ;{ }^{13} \mathrm{C}$ NMR (DMSO- $\left.d_{6}, \delta \mathrm{ppm}\right): 170.3,146.1,143.3$, 141.2, 133.5, 132.7, 131.3, 130.2, 130.2, 129.5, 129.5, 126.7, 123.8, 123.8, 120.1, 108.3, 47.3, 47.3, 11.1; ESI-MS (m/e): $398.11\left(\mathrm{M}^{+}\right)$; Anal. (\%) calcd. (found) for $\mathrm{C}_{19} \mathrm{H}_{15} \mathrm{~N}_{4} \mathrm{O}_{3} \mathrm{SF}$ : C57.28 (57.11), H- 3.79 (3.66), N-14.06 (13.96).

2-(3-Methyl-1-phenyl-1H-pyrazol-4-yl)-3-(4-(trifluoromethyl) phenyl) thiazolidin-4-one (7h): Yield: $65 \%$; m.p. 125-127 ${ }^{\circ} \mathrm{C}$; IR (KBr, $\left.v_{\max }, \mathrm{cm}^{-1}\right): 2927.14\left(-\mathrm{CH}_{3}\right.$, str), 1720.61 (C=O, str), 1321.33 (C-N, str), $1433.24\left(-\mathrm{CH}_{3}\right.$, def), 1160.21 (C-F, str); ${ }^{1} \mathrm{H}$ NMR (DMSO- $d_{6}, \delta$ ppm): 13.7 (s, $\left.1 \mathrm{H},-\mathrm{NH}\right)$, 7.33 (s, 5H), 7.21-7.18 (d, 2H, J=15 Hz, Ar-H), 7.15 (s, 1H, Ar-H), 6.8-6.78 (d, 2H, J = $10 \mathrm{~Hz}, \mathrm{Ar}-\mathrm{H}), 4.81$ (s, 1H, CH-N), 3.43 (s, $\left.1 \mathrm{H}, \mathrm{CH}_{2}-\mathrm{C}=\mathrm{O}\right) ; 3.15$ (s, $\left.1 \mathrm{H}, \mathrm{CH}_{2}-\mathrm{C}=\mathrm{O}\right) ;{ }^{13} \mathrm{C} \mathrm{NMR}$ (DMSO- $d_{6}, \delta$ ppm): $170.3,146.1,143.3,141.2,133.5,132.7$, 131.3, 130.2, 130.2, 129.5, 129.5, 126.7, 124.7, 123.8, 123.8, 120.1, 108.3, 47.3, 47.3, 11.1; ESI-MS (m/e): $403.12\left(\mathrm{M}^{+}\right)$; Anal. (\%) calcd. (found) for $\mathrm{C}_{20} \mathrm{H}_{16} \mathrm{~N}_{3} \mathrm{OSF}_{3}$ : C-59.54 (59.42), H- 4 (3.81), N-10.42 (10.19).
2-(3-Methyl-1-phenyl-1H-pyrazol-4-yl)-3-[4-nitro-3(trifluoromethyl)phenyl]thiazolidin-4-one (7i): Yield: $88 \%$; m.p. $153-154{ }^{\circ} \mathrm{C}$; IR $\left(\mathrm{KBr}, \mathrm{cm}^{-1}\right)$ : $2925.24\left(-\mathrm{CH}_{3}\right.$, str), 1720.61 (C=O, str), $1513.02\left(\mathrm{C}-\mathrm{NO}_{2}\right.$, str), $1436.24\left(-\mathrm{CH}_{3}, \mathrm{def}\right), 1330.21$ (C-F, str), 1321.33 (C-N, str); ${ }^{1} \mathrm{H}$ NMR (DMSO- $d_{6}, \delta$ ppm): 13.1(s, $1 \mathrm{H},-\mathrm{NH}), 8.12-8.10(\delta, 1 \mathrm{H}, \mathrm{J}=10 \mathrm{~Hz}, \mathrm{Ar}-\mathrm{H}), 7.7$ (s, 1H, Ar-H), 7.62-7.60 (d, 1H, J=10 Hz, Ar-H), 7.41 (s, 5H), 7.25 (s, 1H, Ar-H), 2.39 (s, 3H, - $\left.\mathrm{CH}_{3}\right), 4.81$ (s, 1H, CH-N), $3.43\left(\mathrm{~s}, 1 \mathrm{H}, \mathrm{CH}_{2}-\mathrm{C}=\mathrm{O}\right), 3.15\left(\mathrm{~s}, 1 \mathrm{H}, \mathrm{CH}_{2}-\mathrm{C}=\mathrm{O}\right) ;{ }^{13} \mathrm{C}$ NMR (DMSO- $d_{6}, \delta$ ppm): 170.3, 146.1, 143.3, 141.2, 133.5, 132.7, 131.3, 130.2, 130.2, 129.5, 129.5, 126.7, 124.7, 123.8, 123.8, 120.1, 108.3, 47.3, 47.3, 11.1; ESI-MS (m/e): $448.07\left(\mathrm{M}^{+}\right)$; Anal. (\%) calcd. (found) for $\mathrm{C}_{20} \mathrm{H}_{15} \mathrm{~N}_{4} \mathrm{O}_{3} \mathrm{SF}_{3}$ : C-53.57 (53.35), H- 3.53 (3.46), N-12.49 (12.27).

3-(4-Methoxyphenyl)-2-(3-methyl-1-phenyl-1Hpyrazol-4-yl)thiazolidin-4-one (7j): Yield-78 \%; m.p. 137$138{ }^{\circ} \mathrm{C}$; IR $\left(\mathrm{KBr}, v_{\max }, \mathrm{cm}^{-1}\right): 2924.14\left(-\mathrm{CH}_{3}, \mathrm{str}\right), 2855.22$ $\left(-\mathrm{OCH}_{3}\right), 1730.21(\mathrm{C}=\mathrm{O}, \mathrm{str}), 1432.24\left(-\mathrm{CH}_{3}, \mathrm{def}\right), 1321.33$ (C-N, str); ${ }^{1} \mathrm{H}$ NMR (DMSO- $d_{6}, \delta$ ppm): 13.7 (s, $\left.1 \mathrm{H},-\mathrm{NH}\right)$, 7.49 (s, 1H, -N=CH-), 7.37 (s, 5H), 7.21-7.18 (d, 2H, J= 15 $\mathrm{Hz}, \mathrm{Ar}-\mathrm{H}), 7.15$ (s, 1H, Ar-H), 6.8-6.78 (d, 2H, J = $10 \mathrm{~Hz}, \mathrm{Ar}-$ $\mathrm{H}), 4.81$ (s, 1H, CH-N), 3.73 (s, 3H), 3.43 (s, 1H, $\left.\mathrm{CH}_{2}-\mathrm{C}=\mathrm{O}\right)$, $3.15\left(\mathrm{~s}, 1 \mathrm{H}, \mathrm{CH}_{2}-\mathrm{C}=\mathrm{O}\right) ;{ }^{13} \mathrm{C}$ NMR (DMSO- $d_{6}, \delta \mathrm{ppm}$ ): 170.3, 146.1, 143.3, 141.2, 133.5,132.7,131.3, 130.2, 130.2, 129.5, 129.5, 126.7, 124.7, 123.8, 123.8, 120.1, 108.3, 55.1, 47.3, 47.3, 11.1; ESI-MS (m/e): $365.17\left(\mathrm{M}^{+}\right)$; Anal. (\%) calcd. (found) for $\mathrm{C}_{20} \mathrm{H}_{19} \mathrm{~N}_{3} \mathrm{O}_{2} \mathrm{~S}$ : C-65.73 (65.66), H- 5.24 (5.11), N11.50 (11.38).

\section{Pharmacology}

Antioxidant activity: The in vitro antioxidant activity was determined by 1,1-diphenyl-2-picrylhydrazyl radical method, which was used to evaluate the free radical scavenging capacity of different antioxidants ${ }^{11,12}$.

Animals: Wister rat of either sex weighing between 180$200 \mathrm{~g}$ were taken for anti diabetic activity. Animals were maintained under standard environmental condition at temperature of $22 \pm 2{ }^{\circ} \mathrm{C}$ and $45-50 \%$ relative humidity for $24 \mathrm{~h}$ each of dark and light cycle with proper diet. All the studies were done according to protocol approved by Institutional Animal Ethical Committee (IAEC) of Bansal College of Pharmacy (Reg. no-1252/ac/10/CPCSEA, Ref. no-BCP/IAEC/ 12/02).

Acute toxicity study: The acute oral toxicity study was carried out according to OECD guideline no 423 in wister rats $^{13}$. The doses were fixed $2 \mathrm{mg} / \mathrm{kg}$ (p.o) to $10 \mathrm{mg} / \mathrm{kg}$ (p.o) for rats and contain 5 in each group. The mortality and general behaviours were under observation for 14 days. The test compounds were nontoxic in the dose of $5 \mathrm{mg} / \mathrm{kg}$ body weight.

\section{Antidiabetic activity}

Oral glucose tolerance test on rat (OGTT): Twelve groups of animals were administered normal saline at the dose of $5 \mathrm{mg} / \mathrm{kg}$ for test compounds followed by administration of glucose solution in the dose of $2 \mathrm{~g} / \mathrm{kg}$. After $0.5 \mathrm{~h}$ of administration of drug blood sample were withdrawn from dorsal vein at interval of 60,120 and $180 \mathrm{~min}^{14}$. Blood glucose level were estimated using blood glucose test strip with elegance glucometer (Frankenbeng Germany) \& GOD-POD kit (Acuurex, India). 
Induction of diabetes: Streptazocine (STZ) was used in the dose of $60 \mathrm{mg} / \mathrm{kg}$ to induce insulin dependent diabetes. streptazocine was injected into rats intraperitoneally. After 48 $\mathrm{h}$ of administration of streptazocine, the blood samples were collected by dorsal vein for determination of blood glucose level. The rat ${ }^{15}$ with fasting glucose level in range of 275-300 $\mathrm{mg} / 100 \mathrm{~mL}$ - considered as diabetic and considered for study.

Experimental protocol and dose schedule: The total periods for conductance of study were 21 days. The rats were divided into 13 groups consisting of 06 animals in each group.

Group-1: Normal rats treated with normal saline 10 $\mathrm{mL} / \mathrm{kg}$ p.o.

Group-2: Diabetic control treated with streptazocine (60 $\mathrm{mg} / \mathrm{kg}$ ) dissolved in citrate buffer.

Group-3: Diabetic rat treated with rosiglitazone $8 \mathrm{mg} / \mathrm{kg}$.

Group-4-13: Diabetic rat treated with test compounds at $5 \mathrm{mg} / \mathrm{kg}$ body weight.

On 1,7,14 and 21 days of study after $2 \mathrm{~h}$ of oral administration of test compounds blood glucose levels and body weight were measured. Blood samples were withdrawn through dorsal vein. On 21 days whole blood was collected by cardiac puncture. Blood sample collected then centrifuged at $3000 \mathrm{rpm}$ for 10 min after to obtain serum. Blood glucose levels were estimated by GOD-POD kit (Accurex. India). All biochemical parameters were determined. Total cholesterol ${ }^{16}$, triglyceride by Hantzsch condensation method ${ }^{17}$, serum urea and creatinine by method of Thomas ${ }^{18}$, total protein ${ }^{19}$, HDL cholesterol ${ }^{20}$ were measured.

Statistical analysis: The results were shown as Mean \pm SEM and comparison between standard and test compounds were made by one way ANOVA followed by Dunnetts test. Values of $\mathrm{p} \leq 0.001$ were considered as significant.

\section{RESULTS AND DISCUSSION}

The present work involves cyclization of Schiff bases to thiazolidine-4-ones derivatives. The Schiff bases (6a-6j) were obtained by the reaction between electrophilic carbon atom of substituted pyrazole-4-aldehyde and nucleophilic nitrogen atom of substituted amines. The preparation of thiazolidine4-ones (7a-7j) proceeds by an attack of sulphur nucleophile of thioglycolic acid on imine carbon followed by intramolecular cyclization. During reaction one mole of water was eliminated. $\mathrm{SnCl}_{2}, 2 \mathrm{H}_{2} \mathrm{O}$ acted as acid catalyst which counters balance between nucleophilicity and acidity for completion of reaction. The substitution with electron donating group at para and meta position of ring increases percentage yield where as it decreases in case of ortho substitution due to steric effect. Purification of compounds were done using hexane: chloroform $(2: 8 \mathrm{v} / \mathrm{v})$ for thiazolidine-4-ones by column chromatography.

The formations of compounds were again confirmed by elemental analysis (C, H, N analysis). In view of establishment of structure spectral analysis has been performed by IR, ${ }^{1} \mathrm{H}$ NMR, ${ }^{13} \mathrm{C}$ NMR and mass analysis. The IR peaks at 1775.511701.61, 1685-1624, 1321.33-1260.33 and 1290-1200 $\mathrm{cm}^{-1}$ indicate presence of $\mathrm{C}=\mathrm{O}, \mathrm{C}=\mathrm{N}, \mathrm{C}-\mathrm{N}$ groups in synthesized compounds. The characteristic ${ }^{1} \mathrm{H}$ NMR peaks at $\delta$ values 4.85 $4.81,3.49-3.43$ and $3.25-3.15 \mathrm{ppm}$ indicate presence of thiazolidine-4-ones. The aromaticity of the compounds was also confirmed by ${ }^{1} \mathrm{H}$ NMR. The ${ }^{13} \mathrm{C}$ NMR spectral data also describe characteristic peak according to proposed structure. From the mass spectral analysis it was found that $m / e$ peaks according to calculated molecular mass of the synthesized compounds.

Biological evaluation: in vitro Antioxidant activity was determined by DPPH method. The DPPH assay method is based on the reduction of DPPH, a stable free radical. The free radical DPPH with an odd electron gives a maximum absorption at $517 \mathrm{~nm}$. When a solution of DPPH is mixed with that of a substance that can donate a hydrogen atom, then this gives rise to the reduced form with the loss of this violet colour ${ }^{12}$. It is well established that electron donating groups stabilize the resulted phenoxyl radicals through inductive resonance effect; thus lower the $\mathrm{OH}$ bond energy and enhance the radical scavenging activity. In contrary, electron withdrawing groups stabilize more the phenols and destabilize the resulted radicals. In addition, a hydrogen bonding could be formed between the phenoxyl unpaired electron and the adjacent hydroxyl group that stabilizes the radicals formed more than it does for the parent diols. Ascorbic acid is good antioxidant and exhibited high activity reached $99.1 \%$. It could also undergo the two hydrogen-atom transfer process to give the dehyroascorbic acid. Due to this reason the compounds with electron donating groups $\mathbf{7 a}, \mathbf{7 b}, \mathbf{7 d}, \mathbf{7 f}, \mathbf{7 h}$ had shown more $\mathrm{IC}_{50}$ values compare to standard ascorbic acid. Whereas compounds with electron withdrawing groups shown group have shown less $\mathrm{IC}_{50}$ value compare to standard (Table-1).

The acute toxicity study was performed. The study has shown that at $5 \mathrm{mg} / \mathrm{kg}$ body weight the compounds are nontoxic.

\begin{tabular}{|c|c|c|c|c|c|}
\hline \multirow{3}{*}{ Compound code } & \multicolumn{4}{|c|}{$\begin{array}{c}\text { TABLE-1 } \\
\text { OBSERVATION FOR ANTIOXIDANT ACTIVITY IN TERMS OF DPPH METHOD }\end{array}$} & \\
\hline & \multicolumn{5}{|c|}{ DPPH scavenging effect $(\%)$ [mean \pm SEM] } \\
\hline & $25 \mu \mathrm{g} / \mathrm{mL}$ & $50 \mu \mathrm{g} / \mathrm{mL}$ & $75 \mu \mathrm{g} / \mathrm{mL}$ & $100 \mu \mathrm{g} / \mathrm{mL}$ & $125 \mu \mathrm{g} / \mathrm{mL}$ \\
\hline $7 \mathbf{a}$ & $35.61 \pm 0.060$ & $51.31 \pm 0.026$ & $63.41 \pm 0.098$ & $76.97 \pm 0.150$ & $97.32 \pm 0.026$ \\
\hline $7 \mathbf{b}$ & $38.06 \pm 0.010$ & $58.56 \pm 0.060$ & $73.91 \pm 0.050$ & $82.75 \pm 0.120$ & $96.20 \pm 0.140$ \\
\hline 7c & $19.21 \pm 0.120$ & $21.37 \pm 0.090$ & $38.22 \pm 0.240$ & $43.44 \pm 0.150$ & $56.53 \pm 0.160$ \\
\hline $7 d$ & $32.39 \pm 0.050$ & $45.63 \pm 0.025$ & $57.24 \pm 0.160$ & $76.52 \pm 0.052$ & $93.70 \pm 0.010$ \\
\hline $7 e$ & $28.83 \pm 0.098$ & $34.95 \pm 0.026$ & $55.00 \pm 0.023$ & $69.24 \pm 0.050$ & $75.01 \pm 0.110$ \\
\hline 7f & $37.26 \pm 0.250$ & $49.56 \pm 0.130$ & $60.40 \pm 0.160$ & $81.50 \pm 0.090$ & $97.47 \pm 0.120$ \\
\hline $7 g$ & $24.10 \pm 0.130$ & $32.12 \pm 0.250$ & $49.11 \pm 0.120$ & $52.97 \pm 0.010$ & $65.19 \pm 0.110$ \\
\hline $7 \mathrm{~h}$ & $15.00 \pm 0.120$ & $28.23 \pm 0.010$ & $35.31 \pm 0.180$ & $45.01 \pm 0.080$ & $52.11 \pm 0.140$ \\
\hline $7 \mathbf{i}$ & $15.10 \pm 0.170$ & $20.11 \pm 0.050$ & $32.03 \pm 0.200$ & $45.80 \pm 0.030$ & $51.00 \pm 0.080$ \\
\hline $\mathbf{7 j}$ & $28.44 \pm 0.030$ & $47.09 \pm 0.120$ & $51.89 \pm 0.140$ & $75.60 \pm 0.210$ & $96.48 \pm 0.120$ \\
\hline STD (ascorbic acid) & $22.28 \pm 0.120$ & $41.03 \pm 0.190$ & $52.06 \pm 0.200$ & $75.02 \pm 0.090$ & $96.10 \pm 0.180$ \\
\hline
\end{tabular}


The antidiabetic activity was performed by using streptozotocin (STZ) induced model in wister rat. Streptozotocin enters the pancreatic cell via a glucose transporter GLUT2 and causes alkylation of deoxyribonucleic acid. Streptozotocin induces activation of poly adenosine ribosylation and nitric oxide release. Due to destruction of pancreatic cells by streptazocine a huge release of insulin which makes animals more susceptible to severe hypoglycemia that may be lethal. Due to this reason animal treated with streptazocine were administered with $5 \%$ glucose solution for 12-24 h. Afterwards, an increase of glucose levels was observed in comparison to control animals due to insulin deficiency (Table-2) ${ }^{15}$. The thiazolidine were dependent on the presence of insulin for activity. However, they do not affect insulin secretion. The thiazolidine were highly selective and potent agonists for the peroxisome proliferator activated receptor (PPAR) $\gamma$ that regulates the transcription of a number of insulin responsive genes. Activation of PPAR- $\gamma$-receptors regulates the transcription of insulin-responsive genes involved in the control of glucose production, transport and utilization. Additionally, PPAR- $\gamma$ responsive genes also play a role in the regulation of fatty acid metabolism. Unlike oral sulfonylureas, rosiglitazone enhances tissue sensitivity to insulin rather than stimulates insulin secretion ${ }^{21}$. Blood glucose levels in rats administered with $2 \mathrm{~g} / \mathrm{kg}$ glucose were significantly decreased by test compounds with in $1 \mathrm{~h}$ as compared to standard group. Treatment with compounds $\mathbf{7} \mathbf{a}-\mathbf{7} \mathbf{j}$ has shown that there are significant fall of blood glucose level compared to standard rosiglitazone on 21 day of study. But on 21 day of treatment it

\begin{tabular}{|c|c|c|c|c|}
\hline \multicolumn{5}{|c|}{$\begin{array}{l}\text { TABLE-2 } \\
\text { ANTIDIABETIC ACTIVITY LONG TERM EFFECT OF STANDARD ( } 8 \text { MG/KG BODY wt.) } \\
\text { AND COMPOUNDS ( } 5 \text { mg/kg BODY wt.) ON BLOOD GLUCOSE LEVEL OF RAT }\end{array}$} \\
\hline Compound No. & $0 \mathrm{~min}$ & $60 \mathrm{~min}$ & $120 \mathrm{~min}$ & $180 \mathrm{~min}$ \\
\hline \multicolumn{5}{|c|}{ OGTT blood glucose level (mg/dl) } \\
\hline Control & $91.5 \pm 2.23$ & $106.2 \pm 3.02$ & $103 \pm 7$ & $93.67 \pm 1.00$ \\
\hline Rosiglitazone & $91.5 \pm 2.23$ & $110.0 \pm 3.94$ & $108 \pm 1.89$ & $97.83 \pm 0.47 * * *$ \\
\hline $7 \mathbf{a}$ & $93.83 \pm 0.6$ & $152.2 \pm 7.34$ & $132 \pm 0.85$ & $90.83 \pm 0.47 * * *$ \\
\hline $7 b$ & $91.2 \pm 2.21$ & $126.3 \pm 1.23$ & $115 \pm 2.6$ & $93.5 \pm 2.46^{* * *}$ \\
\hline $7 \mathrm{c}$ & $93.83 \pm 0.6$ & $182.7 \pm 1.5$ & $143.3 \pm 3.12$ & $93.5 \pm 2.46$ \\
\hline $7 d$ & $92.2 \pm 1.21$ & $120.1 \pm 1.23$ & $113.2 \pm 2.3$ & $96.5 \pm 2.46^{* *}$ \\
\hline $7 e$ & $90.5 \pm 2.11$ & $126.3 \pm 1.23$ & $110.1 \pm 1.6$ & $93.5 \pm 2.46^{* *}$ \\
\hline $7 f$ & $91.2 \pm 2.21$ & $126.3 \pm 1.23$ & $114.2 \pm 2.6$ & $93.5 \pm 2.46$ \\
\hline $7 g$ & $91.2 \pm 2.21$ & $126.3 \pm 1.23$ & $115.3 \pm 2.6$ & $110.5 \pm 2.46^{* *}$ \\
\hline $7 \mathrm{~h}$ & $96.33 \pm 0.98$ & $191.8 \pm 4.7$ & $149.2 \pm 2.38$ & $95 \pm 1.82 * * *$ \\
\hline $7 i$ & $96.33 \pm 0.98$ & $191.8 \pm 4.7$ & $149.2 \pm 2.38$ & $96.12 \pm 1.82 * *$ \\
\hline $\mathbf{7 j}$ & $92.62 \pm 0.87$ & $153.2 \pm 1.23$ & $130 \pm 2.4$ & $94.3 \pm 2.12 * * *$ \\
\hline Compound No. & 0 days & 7 days & 14 days & 21 days \\
\hline \multicolumn{5}{|c|}{ Fasting blood glucose level (mg/dl) } \\
\hline Control & $91 \pm 1.00$ & $115.7 \pm 3.9$ & $114 \pm 6.8$ & $100 \pm 2.47$ \\
\hline Diabetic control & $244.7 \pm 2.96$ & $281.3 \pm 2.51$ & $311 \pm 3.99$ & $336 \pm 2.12$ \\
\hline Rosiglitazone & $311.3 \pm 3.99$ & $247.8 \pm 7.4$ & $126.7 \pm 5.8$ & $106.5 \pm 1.9$ \\
\hline $7 a$ & $301.7 \pm 3.07$ & $152.5 \pm 1.2$ & $132.2 \pm 1.65$ & $97.83 \pm 0.87 * * *$ \\
\hline $7 \mathrm{~b}$ & $285 \pm 4.8$ & $136.7 \pm 2.04$ & $124.5 \pm 1.60$ & $95.33 \pm 1.33^{* * *}$ \\
\hline $7 \mathrm{c}$ & $301.7 \pm 3.07$ & $123.2 \pm 0.94$ & $119.5 \pm 1.04$ & $112.12 \pm 2.26$ \\
\hline 7d & $311.7 \pm 3.07$ & $120.1 \pm 0.84$ & $116.5 \pm 1.04$ & $105.12 \pm 2.26^{* *}$ \\
\hline $7 e$ & $281.7 \pm 3.07$ & $113.2 \pm 0.93$ & $115.5 \pm 1.04$ & $103.12 \pm 2.26^{* *}$ \\
\hline $7 f$ & $2911.7 \pm 3.07$ & $125.1 \pm 0.92$ & $114.5 \pm 1.04$ & $101.12 \pm 2.26^{* *}$ \\
\hline $7 \mathrm{~g}$ & $312.7 \pm 3.07$ & $124.2 \pm 0.91$ & $113.5 \pm 1.04$ & $105.32 \pm 2.26$ \\
\hline $7 \mathrm{~h}$ & $250.3 \pm 8.51$ & $132.8 \pm 3.19$ & $124.5 \pm 1.29$ & $87 \pm 3.48 * * *$ \\
\hline $7 \mathbf{i}$ & $253.3 \pm 8.51$ & $142.8 \pm 3.19$ & $121.5 \pm 1.29$ & $104 \pm 3.48^{* *}$ \\
\hline $7 \mathbf{j}$ & $286.5 \pm 1.19$ & $193 \pm 3.73$ & $120 \pm 1.89$ & $96.11 \pm 2.26^{* * *}$ \\
\hline \multicolumn{5}{|c|}{ Change in bodyweight (gm) } \\
\hline Control & $160 \pm 0.6$ & $169 \pm 0.5$ & $166 \pm 0.8$ & $163 \pm 0.2$ \\
\hline Diabetic control & $178.5 \pm 0.99$ & $190 \pm 0.69$ & $215 \pm 0.56$ & $253 \pm 0.12$ \\
\hline Rosiglitazone & $171 \pm 0.39$ & $144.5 \pm 0.67$ & $141 \pm 0.60$ & $169 \pm 0.6$ \\
\hline $7 a$ & $168 \pm 0.51$ & $141 \pm 0.6$ & $137 \pm 0.4$ & $162 \pm 0.3 * * *$ \\
\hline $7 b$ & $169 \pm 0.66$ & $132 \pm 0.25$ & $142 \pm 0.7$ & $156 \pm 0.2 * * *$ \\
\hline $7 c$ & $165 \pm 0.6$ & $158 \pm 0.7$ & $143 \pm 0.46$ & $148 \pm 0.2$ \\
\hline $7 d$ & $163 \pm 0.6$ & $148 \pm 0.7$ & $157 \pm 0.41$ & $159 \pm 0.2 * *$ \\
\hline $7 e$ & $162 \pm 0.4$ & $138 \pm 0.7$ & $153 \pm 0.46$ & $161 \pm 0.2 * *$ \\
\hline $7 f$ & $164 \pm 0.3$ & $157 \pm 0.7$ & $153 \pm 0.46$ & $159.1 \pm 0.2 * *$ \\
\hline $7 g$ & $167 \pm 0.2$ & $154 \pm 0.7$ & $153 \pm 0.46$ & $165 \pm 0.2$ \\
\hline $7 \mathrm{~h}$ & $170 \pm 0.21$ & $161 \pm 0.4$ & $155 \pm 0.3$ & $168 \pm 0.2 * * *$ \\
\hline $7 \mathbf{i}$ & $169 \pm 0.21$ & $161 \pm 0.4$ & $153 \pm 0.3$ & $168 \pm 0.2 * *$ \\
\hline $7 \mathbf{j}$ & $168 \pm 0.4$ & $123 \pm 0.61$ & $141 \pm 0.52$ & $159 \pm 0.67 * * *$ \\
\hline
\end{tabular}

Data analyzed by one-way ANOVA followed by Dunnett's test $(n=6) .{ }^{* * *} \mathrm{P} \leq 0.001$ were considered as significant. ${ }^{* *} \mathrm{P} \leq 0.05$ were considered as moderate in activity 


\begin{tabular}{|c|c|c|c|}
\hline $\begin{array}{l}\text { Compound No. group } \\
\text { (mg/kg body wt.) }\end{array}$ & $\begin{array}{l}\text { Cholesterol } \\
(\mathrm{mg} / \mathrm{dl})\end{array}$ & $\begin{array}{l}\text { Triglycerides } \\
(\mathrm{mg} / \mathrm{dl})\end{array}$ & Creati \\
\hline Control & $148 \pm 1.5$ & $83.3 \pm 4.6$ & \\
\hline Diabetic control & $290 \pm 1.9$ & $258 \pm 9.8$ & \\
\hline Rosiglitazone(8) & $119 \pm 2.8$ & $101 \pm$ & \\
\hline $7 \mathbf{a}(5)$ & $136.8 \pm 0.7 * * *$ & $137 \pm 1$ & \\
\hline $7 b(5)$ & $144.5 \pm 0.8 * * *$ & $138 \pm 1^{*}$ & \\
\hline $7 c(5)$ & $143.8 \pm 1.2$ & $123 \pm 1.11$ & \\
\hline & & $123 \pm 1.11 * *$ & \\
\hline $7 e(5)$ & $190.7 \pm 1$ & $111.1 \pm 1.11^{* *}$ & \\
\hline $7 f(5)$ & $142.8 \pm 1$ & $133.2 \pm 1$ & \\
\hline $7 \mathrm{~g}(5)$ & $173.8 \pm$ & $123 \pm 1$ & \\
\hline $7 h(5)$ & $149.8 \pm 1.19 * * *$ & $125.5 \pm 1.6^{* * *}$ & \\
\hline $7 \mathbf{i}(5)$ & $141.1 \pm 1.7^{* *}$ & $131.23 \pm 1.3^{* *}$ & \\
\hline $7 \mathbf{j}(5)$ & $150.5 \pm 2.23 * * *$ & $152 \pm 2.5^{* * *}$ & \\
\hline \multicolumn{4}{|c|}{$\begin{array}{l}\text { Data analyzed by one-way ANOVA followed by Dunnett's test }(n=6) \text {. } \\
\text { moderate in activity }\end{array}$} \\
\hline \multicolumn{4}{|c|}{$\begin{array}{l}\text { was found that there is significant gain of body weight specially } \\
\text { the rat treated with test compound. There was decrease in serum } \\
\text { cholesterol, triglyceride, creatinine, urea levels are decreased } \\
\text { significantly where as the HDL level and total protein levels } \\
\text { are found to increase after } 21 \text { days treatment. But out of all } \\
\text { the compounds tested } \mathbf{7 a}, \mathbf{7 b}, \mathbf{7 h}, \mathbf{7} \mathbf{j} \text { shown more significant } \\
\text { antidiabetic effect against hyperglycemic rats but there is no } \\
\text { significant effect found in case of normoglycemic condition. } \\
\text { Whereas compounds } \mathbf{7 d}, \mathbf{7 e}, \mathbf{7 f}, \mathbf{7 g}, \mathbf{7} \mathbf{i} \text { have shown modeate } \\
\text { activity and compounds } \mathbf{7 c}, \mathbf{7 g} \text { were not significant (Table-3). } \\
\text { From the SAR study it was confirmed that presence of } \\
\text { thiazolidine-4-one nucleus along with pyrazole nucleus is } \\
\text { essential for both antioxidant and antidiabetic activity. But } \\
\text { overall effect depends upon substitution on phenyl ring attached } \\
\text { to thiazolidine nucleus. Compounds with para substitution are } \\
\text { potent, where as compound with para and meta substitution } \\
\text { shown moderate activity. The ortho substituted compounds } \\
\text { are not potent. It were also confirmed that electron withdrawing } \\
\text { substituents at para or meta position decrease the potency of } \\
\text { compounds. }\end{array}$} \\
\hline
\end{tabular}

\section{Conclusion}

The structure of synthesized 2-(3-methyl-1-phenyl-1Hpyrazol-4-yl)-3-phenylthiazolidin-4-ones were confirmed by spectral and elemental analysis. It was found that compounds $\mathbf{7 a}, \mathbf{7 b}, \mathbf{7 h}, \mathbf{7} \mathbf{j}$ are more potent antioxidant and antidiabetic activity compared to standard ascorbic acid and rosiglitazone. This might be due to thiazolidine-4-one nucleus present along with pyrazole ring. It was also found that phenyl ring substitution have great effect on biological activity. In compounds, the substitutions on phenyl ring at para position with electron donating groups have shown potent activity compare to standards.

\section{ACKNOWLEDGEMENTS}

The authors acknowledge to IIMT College of Medical Sciences; Regional Institute of Pharmaceutical sciences and Technology, Govt of Tripura; AIRF, JNU, New Delhi and Arbro
Lab, New Delhi. The authors also wish to acknowledge Arunabaha Mallik, Assistant Professor, Bansal College of Pharmacy, Bhopal for providing the facility for animal study.

\section{REFERENCES}

1. E.M. Sharshira and N.M.M. Hamada, Molecules, 17, 4962 (2012)

2. M.R. Chaurasia, Indian J. Pharm., 33, 17 (1971).

3. B. Zimenkovsky, R. Lesyk, O. Vladzimirska, I. Nektegayev, S. Golota and I. Chorniy, J. Pharm. Pharmacol., 51, 264 (1999).

4. A.R. Saltiel and J.M. Olefsky, Diabetes, 45, 1661 (1996).

5. S. Schwartz, P. Raskin, V. Fonseca and J.F. Graveline, N. Engl. J. Med., 338, 861 (1998).

6. J. Buse, B. Gumbiner, N.P. Mathias, D.M. Nelson, B.W. Faja, R.W. Whitcomb, The Troglitazone Insulin Study Group, Diabetes Care, 21, 1455 (1998).

7. D. Quilliot, E. Walters, J.-P. Bonte, J.-C. Fruchart, P. Duriez and O. Ziegler, Am. J. Clin. Nutr., 81, 1117 (2005).

8. B.S. Furniss, A.J. Hannaford, P.W.G. Smith and A.R. Tetchell, Vogels Text Book of Practical Organic Chemistry, Longman Ltd., England, edn. 5, pp. 243-244, 1150 (1998).

9. R.M. Ambika Srivastava Singh, Indian J. Chem., 44B, 1868 (2005).

10. Y. Li, Z.S. Yang, H. Zhang, B.J. Cao, F.D. Wang, Y. Zhang, Y.-L. Shi, J.-D. Yang and B.-A. Wu, Bioorg. Med. Chem., 11, 4363 (2003).

11. W. Brand Willams, M.E. Cuvelier and C. Berset, Lebensm.-Wissen. Technol., 28, 25 (1995).

12. J.C. Espin, C. Soler Rivas and H.J. Wichers, J. Agric. Food Chem., 48, 648 (2000)

13. OECD, Guidelines No. 423 for the Testing of Chemicals Revised Draft Guideline 423 Acute Oral Toxicity, Paris (2000).

14. S.V. Kadnur and R.K. Goyal, Indian J. Pharm. Sci., 67, 453 (2005).

15. T.S. Frode and Y.S. Medeiros, J. Ethnopharmacol., 115, 173 (2008).

16. N. Rifaci, P.S. Bachorik and J.J. Albers, in eds.: In C.A. Burtis and E.R. Ashwood, Lipid, Lipoprotein and Apolipoprotein, Tretz Text Book of Clinical Chemistry, WB Saunder Company, Philadolphia, edn. 3, pp. 809-861 (1999).

17. R.P. MacDonald, Standard Methods of Clinical Chemistry, Academic Press, New York, pp. 215-222 (1953).

18. L. Thomas, Clinical Laboratory Dragnostics, TH Book Verlasgesell schaft, Frankfurt, pp. 208-214, 366-374 (1998).

19. N.W. Tretz, Text Book of Clinical Chemistry, WB Saumder Company, Philadelphia, edn. 3, p. 579 (1986).

20. M. Burstein, H.R. Scholnick and R. Morfin, J. Lipid Res., 11, 583 (1970).

21. M. Imran, B. Ilyas and S.A.K. Deepanjali, J. Sci. Ind. Res., (India), 66, 99 (2007). 